CORRIGENDUM

doi:10.1038/nature09382

\title{
Fighting the monster
}

Amy Maxmen

Nature 466 (suppl.), S18-S19 (2010)

This Outlook article incorrectly stated that David McMurray is not affiliated with Aeras; in fact he is on the board of Aeras, but he is not directly working on the AERAS-402 vaccine. 